\title{
Nano-Bio-Interaction of Gold Nanoparticles with Cancer Cells and Impacts on Biophysical Properties
}

\author{
Ahmad Sohrabi Kashani', Simona Badilescu', Alisa Piekny ${ }^{2}$ and Muthukumaran Packirisamy ${ }^{1}$ \\ ${ }^{1}$ Optical Bio Microsystem Lab., Mechanical, Industrial and Aerospace Engineering Department, Concordia University \\ 1455 De Maisonneuve Blvd, W, Montreal, Quebec, Canada \\ ah_sohra@encs.concordia.ca; simonabadilescu0@gmail.com; mpackir@encs.concordia.ca \\ ${ }^{2}$ Biology Department, Concordia University \\ 7114 Sherbrook Street W., Montreal, Quebec, Canada \\ alisa.piekny@concordia.ca
}

\section{Extended Abstract}

Despite many advancements in detection and treatment, cancer remains the main cause of death in the world. During the cancer progression, the functional behaviour of cells such as adhesion, invasion, migration, and signalling are altered [1]. These changes can be accompanied by the alteration of the biophysical properties of cells. It has been substantiated that there is a correlation between the metastasis potential of cells and their mechanobiological properties. The stiffness of cancer cells is reduced compared to their healthy counterparts, enabling them to pass through endothelial cells, enter into the circulation system and invade the neighbouring tissues. This process, the metastasis, is the main reason for the deaths due to cancer [2]. The mechanical properties of cells are determined by the cytoskeletal structure and during the cancer progression, this structure is reorganized, enabling cancer cells to change from immotile state to motile state[3][4]. Nanotechnology has provided a great platform to develop nano-tools for detection and treatment of cancer. With the help of nanotechnology, nanoparticles can be designed to target the cytoskeletal proteins to regulate the metastasis level of cancer cells. Direct or indirect interaction of nanoparticles with filamentous proteins affects the structural remodelling of cancer cells and ultimately can hamper or reduce the dynamical capacity of cells. Due to the disturbing influence of nanoparticles, the migration and motility ability of cells can be changed[5][6].

In the current work, we have studied the interaction of two different gold nanoparticles (nanosphere and nanostar-shaped) with lung human cancer cells (A549) to investigate their cytotoxicity as well as their impacts on the cellular mechanobiology. The two types of Au nanoparticles were synthesised by chemical methods [7] [8] by reduction of a gold precursor and characterized with different techniques: Scanning Electron Microscopy (SEM), UV/Visible spectroscopy and Light Dynamic Scattering (DLS). SEM and DLS measurements showed that the average diameter of nanospheres and nanostars are $\sim 22 \mathrm{~nm}$ and $\sim 80 \mathrm{~nm}$ respectively. Gold nanoparticles appeared to be non-toxic to the cells as no cell death was observed; however, some disruptions have been seen in the cytoskeletal structure of cells. Atomic Force Microscopy (AFM) and scratch assays were utilized to characterize the stiffness and the migration ability of cells upon interaction with the gold nanoparticles. The results showed that the stiffness and migration ability of A549 cells are influenced by nanoparticles. However, the level of the effect depends on the shape and size of nanoparticles. Our results showed that the stiffness of cells is reduced by $50 \%$ after interaction with gold nanoparticles, confirming their potential to significantly change the cellular mechanobiology. The results showed that star-shaped particles are less effective in changing the mechanobiological properties of cancer cells, likely due to their shape and size. These preliminary data illustrate the potential applications of gold nanoparticles in inhibiting cancer metastasis. 


\section{References}

[1] E. Moeendarbary and A. R. Harris, "Cell mechanics: Principles, practices, and prospects," Wiley Interdiscip. Rev. Syst. Biol. Med., vol. 6, no. 5, pp. 371-388, 2014.

[2] Y. Nematbakhsh and C. T. Lim, "Cell biomechanics and its applications in human disease diagnosis," Acta Mech. Sin., vol. 31, no. 2, pp. 268-273, 2015.

[3] D. A. Fletcher and R. D. Mullins, "Cell mechanics and the cytoskeleton," Nature, vol. 463, no. 7280, pp. 485-492, 2010.

[4] A. S. Kashani, S. Badilescu, A. Piekny, and M. Packirisamy, "Bio-Nano-Interaction in Treatment and Management of Cancer," Journal of the Electrochemical Soceity, vol. 166, no. 9, pp. 3007-3011, 2019.

[5] K. Buyukhatipoglu and A. M. Clyne, "Superparamagnetic iron oxide nanoparticles change endothelial cell morphology and mechanics via reactive oxygen species formation," J. Biomed. Mater. Res. - Part A, vol. 96 A, no. 1, pp. 186-195, 2011.

[6] D. Septiadi, F. Crippa, T. L. Moore, B. Rothen-Rutishauser, and A. Petri-Fink, "Nanoparticle-Cell Interaction: A Cell Mechanics Perspective," Adv. Mater., vol. 30, no. 19, pp. 1-30, 2018.

[7] N. Anand, S. Venkatesh, P. Putta, S. Stoenescu, S. Badilescu, M. Packirisamy, V. Truong., "Fabrication of a Gold Nanostar-Embedded Porous Poly (dimethylsiloxan) Platform for Sensing Applications," Sensors \& Transducers, vol. 149, no. 2, p. 20, 2013.

[8] D. Beaudet, S. Badilescu, K. Kuruvinashetti, A. S. Kashani, D. Jaunky, S. Ouellette, A. Piekny, M. Packirisamy, "Comparative study on cellular entry of incinerated ancient gold particles (Swarna Bhasma) and chemically synthesized gold particles," Sci. Rep., vol. 7, no. 1, p. 10678, 2017. 